\title{
Automatic vessel monitoring with single and multidimensional SAR images in the wavelet domain
}

\author{
Mariví Tello ${ }^{1}$, Carlos López-Martínez ${ }^{*}$, Jordi J. Mallorqui ${ }^{2}$ \\ Universitat Politecnica de Catalunya, Barcelona, Spain \\ Signal Theory and Communications Department, Campus Nord Polytechnic University of Catalonia, \\ UPC 1-3, Jordi Girona 08034, Barcelona, Spain
}

Received 28 March 2006; received in revised form 18 September 2006; accepted 18 September 2006

Available online 13 November 2006

\begin{abstract}
Spaceborne Synthetic Aperture Radar (SAR) techniques constitute an extremely promising alternative compared to traditional surveillance methods thanks to the all-weather and day-and-night capabilities of Radar linked with the large coverage of SAR images. Nowadays, the capabilities of satellite based SAR systems are confirmed by a wide amount of applications and experiments all over the world. Nevertheless, specific data exploitation methods are still to be developed to provide an efficient automatic interpretation of SAR data. The aim of this paper is to present an approach based on multiscale time-frequency analysis for the automatic detection of spots in a noisy background which is a critical matter in a number of SAR applications. The technique has been applied to automatic ship detection in single and multidimensional SAR imagery and it has proven to be a rapid, robust and reliable tool, able to manage complicated heterogeneous scenes where classical approaches may fail.

(C) 2006 International Society for Photogrammetry and Remote Sensing, Inc. (ISPRS). Published by Elsevier B.V. All rights reserved.
\end{abstract}

Keywords: Vessel monitoring; Synthetic aperture radar; Polarimetry; Time-frequency analysis; Wavelet transform

\section{Introduction}

Nowadays, there is an increasing interest in remote sensing applications which can be critically helpful to guarantee a better understanding, monitoring and predicting of the Earth's global climate change, as well as to the management of natural resources, disasters and human activities. In Europe, GMES (Global Monitoring for Environment and Security), which is a

\footnotetext{
* Corresponding author. Tel.: +34 934016785.

E-mail addresses: marivi.tello@tsc.upc.edu (M. Tello), carlos.lopez@tsc.upc.es (C. López-Martínez),mallorqi@tsc.upc.edu (J.J. Mallorqui).

${ }^{1}$ Tel.: +34934017426.

2 Tel.: +34934017229.
}

joint endeavour of the European Space Agency and the European Community remains a valuable proof of the actual rise of space technologies (Cornaert et al., 2001).

One important field of application is maritime monitoring from space, which includes among others traffic surveillance, maritime security, fisheries and border control (Greidanus, 2005). For example, carrying out an effective control of fishing activities is essential to guarantee a sustainable exploitation of sea resources: illegal fishing can lead to over-exploitation, catches over safe biological limits and disturbances to the fisheries habitat. It is also crucial to detect smuggling and illegal immigration movements. As the regulated areas are extended, they are difficult to monitor by means of traditional reconnaissance methods such as planes or 
patrol vessels. Spaceborne SAR sensors are able to provide a powerful surveillance capability allowing the observation of broad expanses, independent of weather effects, so much during the day as during the night and they do not require any kind of active cooperation of the vessel. For instance, since January 1 st of 2005, all European Community fishing vessels exceeding $15 \mathrm{~m}$ overall length are subject to have installed on-board a VMS for controlling purposes. The Vessel Monitoring System (VMS) (Vessel Monitoring System (VMS) Information Website: http://www.glf.dfo-mpo.gc.ca/ $\mathrm{fm}-\mathrm{gp} / \mathrm{cp}$-cp/vms-ssn/index-e.jsp), is basically an onboard transponder that provides vessel identification and position via satellite communications. Although this transponder-based monitoring system provides accurate results it is not perfectly suitable for monitoring illegal activities as the hardware can be intentionally disconnected or damaged. A complementary or even alternative solution is provided by SAR imagery. Aware of this problem the European Community has promoted the DECLIMS concerted action that brought together 24 parties active in space-based ship detection. The project provided a focus for research into the use of satellite imagery for maritime vessel detection, classification and identification, evaluate and compare the performances of the different algorithms, strengthen the infrastructure capable of meeting demands of users and set the requirements of new sensors and platforms towards the operational needs of vessel monitoring (Greidanus et al., 2004).

It is widely known that the human vision, as it can perceive a structure in the context of its surroundings, can manage non stationarities in a convenient way, overcoming existing automatic algorithms in extracting features in a complex scene. For example, some vessels undetected by conventional techniques in SAR images are clearly visible by eye. Nevertheless, since a manual treatment is unacceptably slow, unpractical and hardly reproducible, fully or at least partially automatic schemes are desirable. The main concern of this paper is to reproduce the behaviour of the human vision by means of timefrequency methods to provide efficient techniques based on this framework for automatic ship detection purposes.

The paper is structured as follows. First, the statistical characterization of SAR images is presented in order to highlight the complexity of automatic ship detection in this context. Section 4 details and analyzes the advantages of using multiscale time-frequency methods for the interpretation of SAR data. Section 5 introduces and analyzes a specific technique for automatic ship detection, based on the wavelet transform. The performances of the algorithm are tested on ENVISAT and
RADARSAT images, which are validated on the basis of available ground truth. An extension to multidimensional SAR data will be introduced and employed to discuss the benefits of polarimetric diversity for automatic ship detection. The last section of this paper presents the conclusion of the work.

\section{Particularities of SAR imagery}

A complex SAR image $u(x, r)$ may be modelled as the convolution of the local complex reflectivity of the observed area $\gamma(x, r)$ with the impulse response of the SAR system $u_{0}(x, r)$ (Curlander and McDonough, 1991). The reflectivity for each resolution cell results from the complex sum of the contributions of all individual scatterers within it $\gamma_{i}(x, r)$

$|u(x, r)|=\left|\gamma(x, r) * u_{0}(x, r)\right|=\left|\sum_{i} \gamma_{i} * u_{0}(x, r)\right|$

where $x$ and $r$ represent the spatial dimensions of the SAR image known as azimuth and range, respectively. This complex sum, also called random walk process, produces speckle (Goodman, 1976) (Lee, 1981), which represents one of the major disturbances for the correct interpretation of SAR images. Due to the complexity of the electromagnetic scattering process, speckle must be considered as a noise source, characterized by being spiky, with a large dynamic range and, contrarily to optical imagery, making SAR images not suggestive to inexperienced end users. In case of homogeneous areas, the most commonly used model to describe SAR images is that of the multiplicative speckle noise, which expresses the observed intensity $I(x, r)$ as the product of a deterministic-like radar cross section $\sigma^{2}(x, r)$ by the speckle noise $n(x, r)$

$I(x, r)=|u(x, r)|^{2}=\sigma^{2}(x, r) n(x, r)$.

The speckle noise component $n(x, r)$ presents an exponential distribution

$P_{n}(n)=\exp (-n) n \in[0, \infty)$

which is characterized by a mean and a variance values equal to one.

Consequently, analysis tools have to be embedded in a statistical framework, since SAR image samples are realizations of some underlying random variable. According to the central limit theorem, the statistical characteristics of a discrete signal can be estimated through the values of the signal in a cluster of samples. And the more extended the cluster, the more accurate the estimation of their statistical properties. Nevertheless, the Heisenberg 
principle states that this is necessarily obtained at the expense of a loss of spatial resolution. Since SAR images represent non stationary phenomena and, since the location information is crucial in most of the applications, the spatial resolution cannot be completely sacrificed for an accurate estimation of statistical (or frequency) parameters. This trade off justifies the use of a suitable time-frequency framework for the interpretation of SAR data in order to estimate the statistical information minimizing the loss of spatial resolution or spatial details.

\section{Automatic spot detection: main drawbacks}

In order to develop a novel framework based on multiscale time-frequency methods for the analysis and automatic exploitation of SAR data, the first canonical problem to treat is spot detection. A spot may be defined as a small distinct area that is different in colour, material or texture from its surroundings. This paper will be treating ship detection in spaceborne SAR images, but spot detection is also present in a large number of very diverse domains, such as in medical applications (e.g. the detection of micro calcifications in mammograms (Zheng et al., 1996)), in astronomy (stars tracking (Liebe, 1993)) and of course in different remote sensing applications (detection of permanent scatterers (Ferreti et al., 2001), cars tracking, etc.). These cases can be seen in Fig. 1. The intuitive and common sense definition of spot implies a quantitative determination of the notion of difference in a computerized tool to detect spots.

Due to the great diversity of types of vessels, the wide range of image modes and resolutions and the distortions affecting the signatures of the ships, a priori information about size or shape cannot be employed to assist the detection. In fact, the low resolution of the sensors and the different motion distortions render the ship's signature rather featureless, since the same ship can present very different signatures depending on a
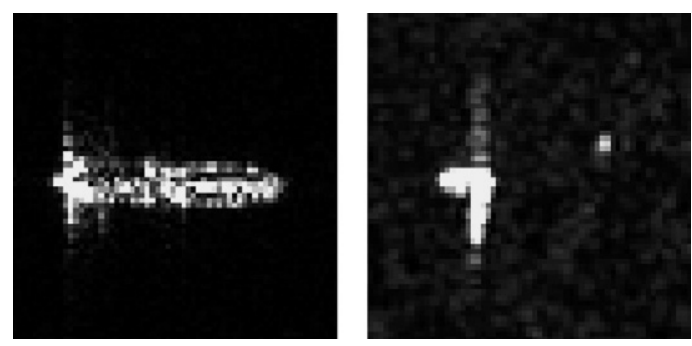

Fig. 2. Left: ship in a high resolution SAR image. Right: two ships in a low resolution SAR image.

number of unpredictable random variables, mainly related to sea state, see Fig. 2.

Nowadays, several systems present operational status for automatic ship detection purposes on SAR imagery. All of them rely on a Constant False Alarm Rate (CFAR) approach which interprets the difference between the spot and its surrounding area as a difference of intensity levels (Arnesen and Olsen, 2004), see Fig. 3.

The underlying reasoning associated to CFAR techniques is valid and efficient if the observed scene is sufficiently stationary but it fails elsewhere. Essentially, these algorithms assume the homogeneity of the statistical distribution of the image to be analyzed within a region of fixed dimensions. Through the values of the signal in this cluster of samples, some statistical parameters are estimated. They are employed to adjust the histogram to the probability density function (pdf) of the particular statistical distribution previously assumed to characterize clutter. Then, a threshold is calculated and its application leads directly to a binarized image. Hence, summing up, the validity of CFAR approaches strictly depends on two conjectures: the assumption of a particular nature of statistical distribution and the hypothesis that a considerable contrast exists between the vessel to be detected and the surrounding sea. Nevertheless, these requirements are not always met, as

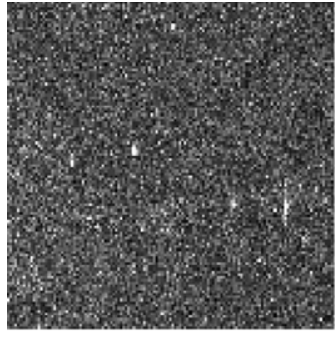

a)

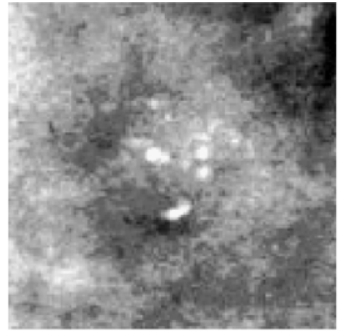

b)

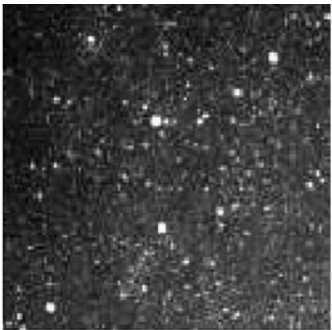

c)

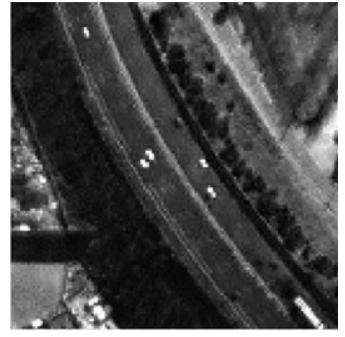

d)

Fig. 1. Different scenarios involving spot detection. (a) Ships on a SAR image, (b) micro calcifications in a mammogram, (c) stars tracking, (d) cars in an optical image from IKONOS. 


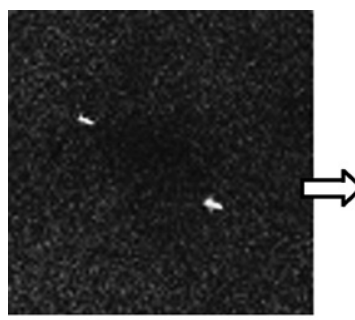

SAR image

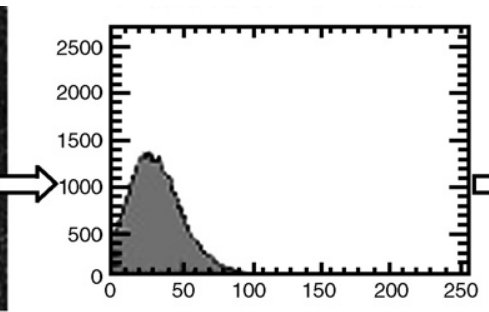

Histogram

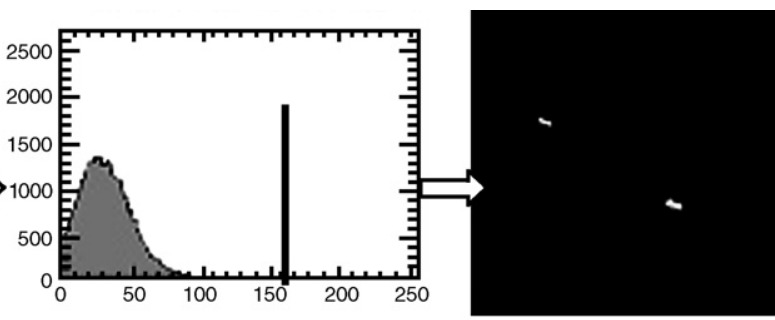

Thresholding

Result

Fig. 3. Flow chart of the basic steps of CFAR algorithms.

it can be observed in two different examples shown in Figs. 4 and 5.

An interesting observation that arises from different experiments carried out to check the feasibility of detection of marine traffic from space is that existing software based on CFAR algorithms is not as good as a human operator, who is better at dealing with complex clutter situations (Greidanus, 2006). Inspired on the human vision operation, the objective of the method proposed in this paper is to provide an alternative approach to CFAR techniques. Besides taking exclusively an image's intensity characteristics for spot detection into account, our method focuses also on a spot's very local statistical behaviour. More specifically, the eye is an extremely sophisticated system which can perceive local regularity, seeing a texture over two fundamental properties: the orientation of its elements and its frequency content. From a signal processing point of view, this translates into performing a timefrequency analysis. Moreover, the human vision can also manage contextual information thanks to its capability of focusing and relating elements at different scales. In signal processing terms, this means performing a multiresolution analysis. This paper proposes to mimic this operation taking advantage of the Wavelet Transform (WT) properties (Mallat, 1999).

\section{The wavelet transform}

The WT addresses the study of a complex phenomenon by dividing it into different simpler pieces. Mathematically, this means projecting a given function in a function space in which the simple pieces are the elements of the basis. The particularity of the wavelet function space is that the basic functions or atoms $\psi_{j}$ are localized in both time and frequency. Moreover, they
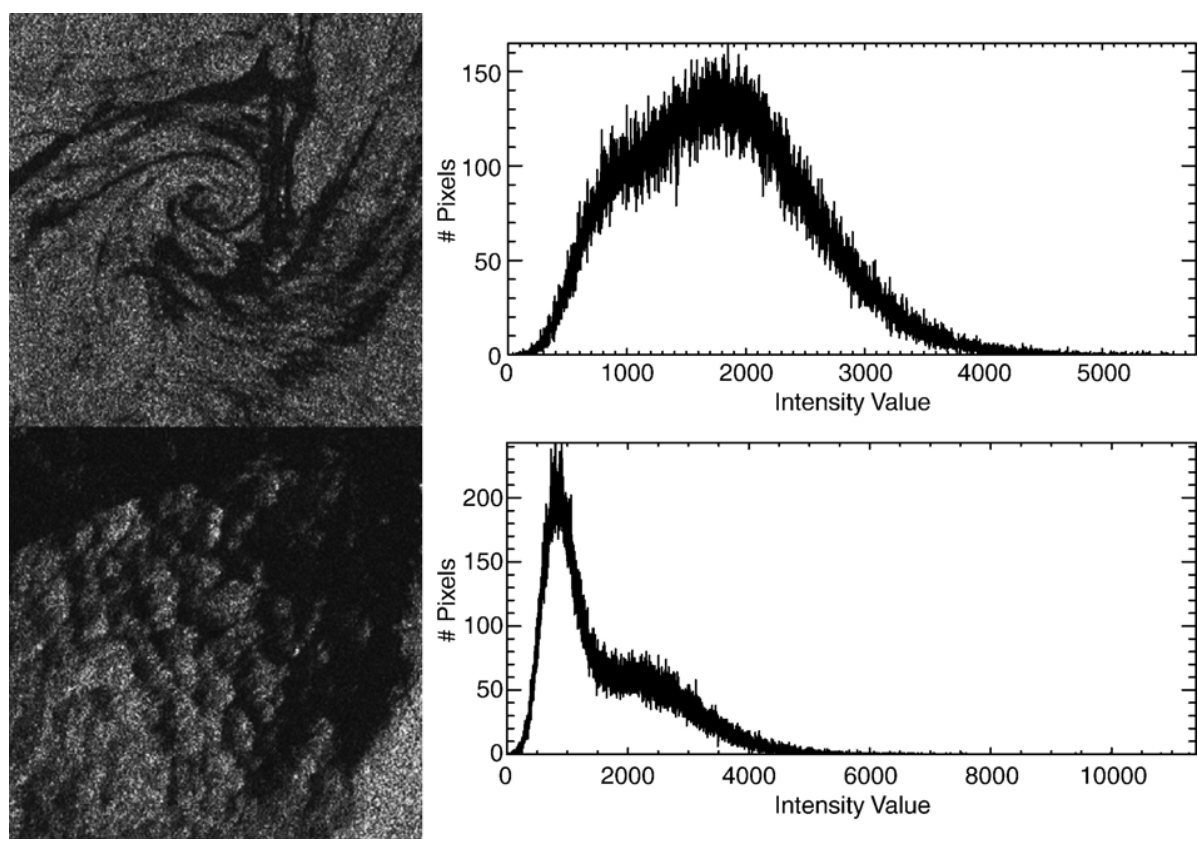

Fig. 4. Two examples of heterogeneous oceanic scenes with their respective histograms. 

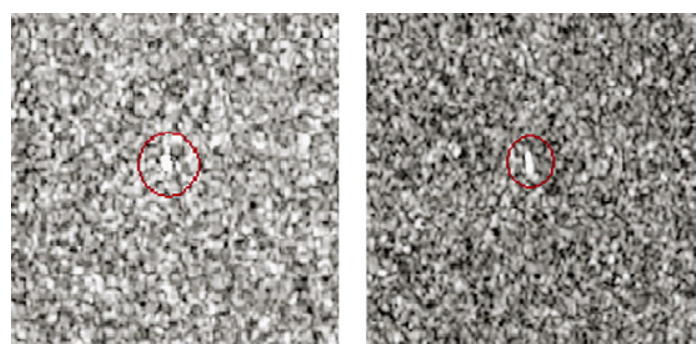

Fig. 5. Two examples of ships signatures (enclosed in a circle) exhibiting a low contrast respect to the background sea surface.

come from dilations and translations of a mother wavelet, $\psi$ (Mallat, 1999)

$\psi_{j}[z]=\frac{1}{\sqrt{a^{j}}} \psi\left[\frac{z}{\mathrm{a}^{j}}\right]$.

Hence, the WT is defined as

$$
W f\left[z, \mathrm{a}^{j}\right]=\sum_{m=0}^{N-1} f[m] \psi_{j}^{*}[m-z] .
$$

The WT is an iterative process where $z$ is the discrete temporal (or spatial) variable, $j$ represents the number of iterations, the parameter $a^{j}$ allows adjusting the temporal (or spatial) duration of the wavelet $\psi_{j}$. Nevertheless, according to the Heisenberg uncertainty principle stating that there is a trade off between time and frequency accuracies, the surface of the wavelet atoms in the time-frequency space is low bounded

$\sigma_{t}^{2} \sigma_{\omega}^{2} \geq \frac{1}{4}$

where $\sigma_{t}$ and $\sigma_{\omega}$ are the temporal and frequency variances. Hence, the resolutions in time and in frequency cannot be arbitrarily refined. Moreover, if a finer resolution in time or space is required the counterpart, the resolution in frequency, is degraded.

The WT can also be seen as a multiscale differential operator. More specifically, a continuous wavelet $\psi$ with a fast decay presents $n$ vanishing moments if and only if there exists a function $\theta$ with a fast decay such that

$\psi(t)=(-1)^{n} \frac{\mathrm{d}^{n} \theta(t)}{\mathrm{d} t^{n}}$

where $t$ stands for the continuous temporal variable. As a consequence,

$W f(u, s)=s^{n} \frac{\mathrm{d}^{n}}{\mathrm{~d} u^{n}}\left(f * \bar{\theta}_{s}\right)(u)$ where $s$ is the scale, $u$ denotes the time variable and $\bar{\theta}_{s}$ is the conjugate of the corresponding wavelet at scale $s$, constructed from a dilation of the function $\theta$.

Signal singularities may be detected by finding the abscissa where the wavelet modulus maxima converge at fine scales (Mallat, 1999). For example, in the particular case in which the wavelet has only one vanishing moment, wavelet modulus maxima are the maxima of the first order derivative of $f(t)$ smoothed by $\bar{\theta}_{s}$. This property can be considered for automatic spot detection purposes since spots and for instance, ships in SAR images - can be regarded as isolated (since they occupy a finite number of points) cusps (i.e., a non oscillating singularity in which the function or one of its derivatives approaches infinity at a certain point).

In $2 \mathrm{D}$, the discrete WT is usually employed separately to each dimension applying a filter bank. In this paper, we will use the Over Complete WT (OCWT) (Law and Siu, 2003) which is a variation from the basic sub-sampling representation provided by the WT, aiming to achieve translation invariance. The OCWT is a process consisting on iteratively applying lowpass $H$ and bandpass $G$ filters to both horizontal and vertical directions of the input signal $X$ in such a way that three components of Eq. (9) are obtained at each iteration as it can be observed in Fig. 6. Intuitively, when a bandpass filter is being applied only in the horizontal dimension of an image, fast changes in this direction, i.e. vertical boundaries, are being enhanced. The same is true in the other dimension. As a consequence, $D_{j}^{1}$ detects vertical edges in an image, whereas $D_{j}^{2}$ does the same for horizontal ones.

$X_{j}\left(z_{1}, z_{2}\right)=H\left(z_{1}^{j}\right) H\left(z_{2}^{j}\right) X_{j-1}\left(z_{1}, z_{2}\right)$

$D_{j}^{1}\left(z_{1}, z_{2}\right)=G\left(z_{1}^{j}\right) X_{j-1}\left(z_{1}, z_{2}\right)$

$D_{j}^{2}\left(z_{1}, z_{2}\right)=G\left(z_{2}^{j}\right) X_{j-1}\left(z_{1}, z_{2}\right)$

Where $z_{1}$ is the horizontal dimension, $z_{2}$ is the vertical one.

In order to present the underlying intuition of the algorithm, it may be interesting to observe the effects

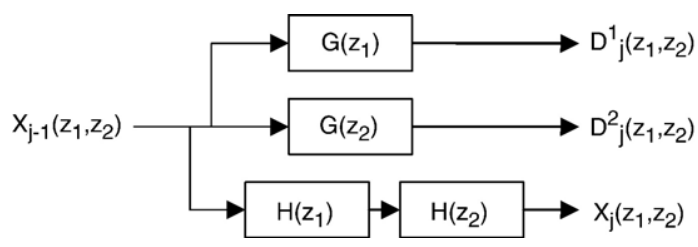

Fig. 6. Flow chart of one iteration of the OCWT. 


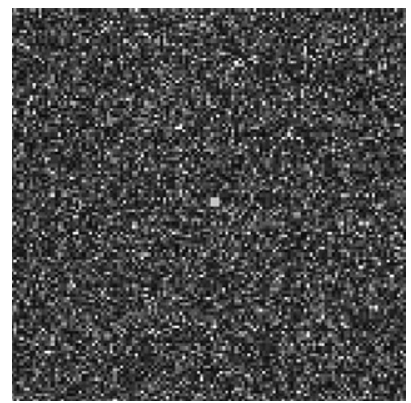

Fig. 7. Simulated image with a small $(2 \times 2 \mathrm{px}$. $)$ and weak target embedded in a noisy background.

of the application of the OCWT on a simulated image in which a small and weak structure has been embedded in a noisy background, see Fig. 7. Since the intensity of the structure is not the maximum value in the image, the situation presented would not be resolvable by CFAR approaches, even if the spot is discernible by eye.

In signal theory, it is quite intuitive that the difference between noise and a structure is the spatial correlation. More specifically, spatial correlation in the noise is low, i.e., pixels are usually not related to each other, and it is quite unlikely for a pixel to have a similar intensity than its neighbours. On the contrary, spatial correlation on a structure is high, i.e., the probability that a pixel has similar intensity as its neighbours is high. When applying the OCWT, this different behaviour is transferred to the wavelet domain much more amplified because the WT is an effective whitening process, see

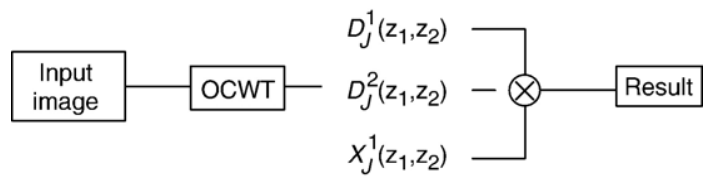

Fig. 9. Flow chart of the proposed algorithm for automatic spot detection.

Fig. 8. In fact, Flandrin (1992) showed that within-scale wavelet coefficients are uncorrelated for Fractional Brownian Motion (FBM) (which is the traditional model assumed for oceanic sea surface turbulences) (Stewart et al., 1993). Moreover, since the WT enhances the presence of singularities, the probability of coincidence of local maxima in the different subbands within a scale is low in the background noise, whereas the probability of co-occurrence of local maxima is high in the presence of a structure.

\section{Automatic spot detection with single channel SAR data}

\subsection{Theoretical principles}

Based on the previous hypothesis and taking advantage of the difference of behaviour of the noise and the target in the wavelet domain, a novel approach for ship detection in SAR imagery is proposed, see Fig. 9. This algorithm consists of two operations per iteration. The first one is the application of a single iteration of the OCWT which leads to three different
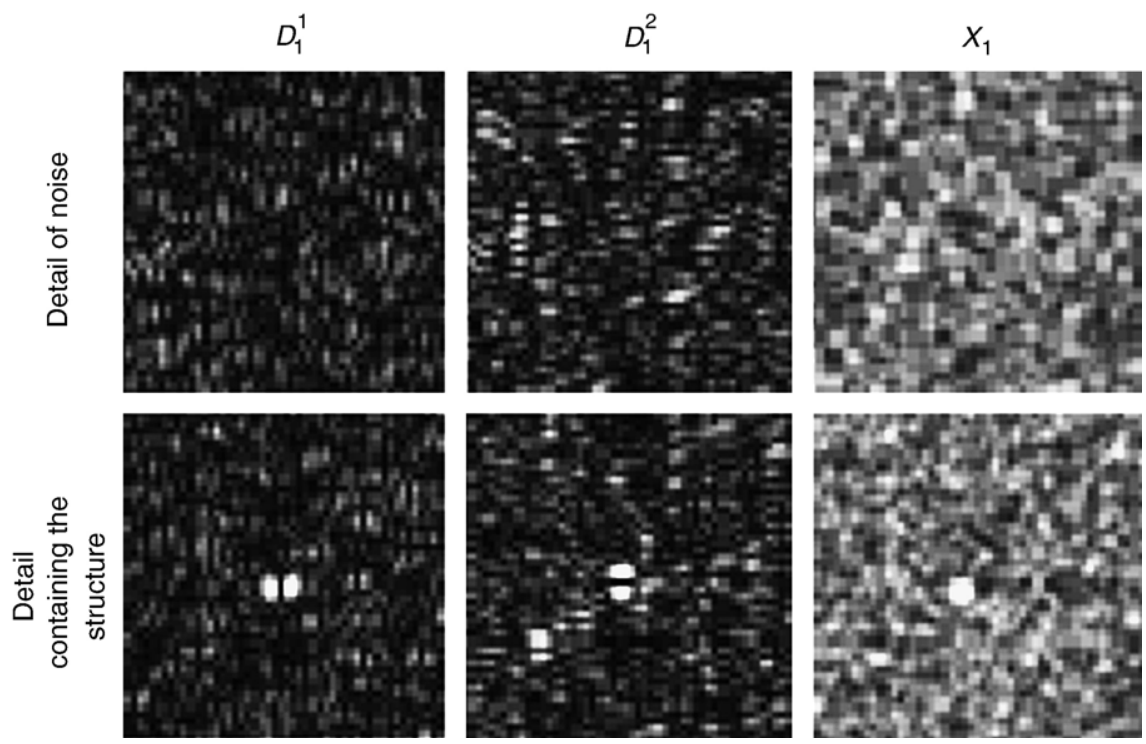

Fig. 8. Detail of the wavelet coefficients for a zoom on two different regions of the simulated image. 


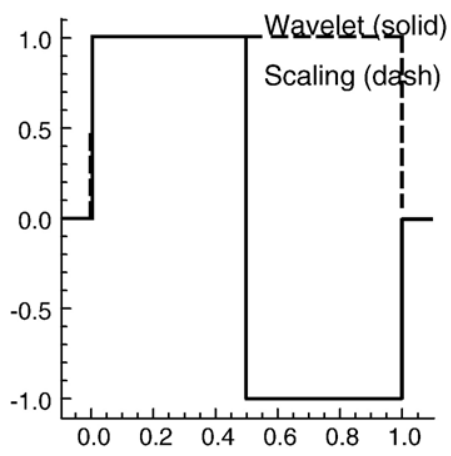

Fig. 10. Haar wavelet and corresponding scaling function.

components $D_{j}^{1}, D_{j}^{2}$ and $X_{j}$ as presented before. Then, the second one consists of merging the three components into one result by a spatial product. The spatial product accounts for the different behaviour of the noise and structural information and privileges co-occurrence of local maxima in the wavelet subbands. As a result, the presence of the structure is enhanced with respect to clutter. This basic step can be iterated by applying it to the wavelet subband $X_{j}$ corresponding to a low pass filtered version of the input image.

There exist different wavelet bases (Villasenor et al., 1995). In fact, a wavelet family can be constructed through dilations and translations of a mother wavelet which can be any function $\psi \epsilon L^{2}{ }^{\circledR}$ satisfying the admissibility condition, i.e. having zero average

$\int_{-\infty}^{+\infty} \psi(t) \mathrm{d} t=0$

Then, the difference between mother wavelets relies on two properties: the number of vanishing moments of $\psi$ and size of its support. On the one hand, it has been seen before that a WT can be though of as a multiscale differential operator. The number of vanishing moments of the mother wavelet is related to the order of the derivative (Mallat, 1999). On the other hand, the size of the compact support of the wavelet is the number of non-zero coefficients in time or space. According to these criteria for the selection of the wavelet as well as to the type of application focused, the Haar basis is considered in the following, see Fig. 10. First, the Haar wavelet has the shortest support among all orthogonal wavelets. The size of the support is critical for localization purposes involved in ship detection. The Haar wavelet has only two non-zero coefficients at $n=0$ and $n=1$. Second, it has only one vanishing moment, i.e., it can be considered as a multiscale differential operator of order 1. In that sense, it can be treated as the Canny edge detector, not well localized in frequency and therefore not well suited to approximate smooth functions, but quite appropriate to spot detection.

For the number of iterations and according to the span of resolutions of space-borne sensors that are operational nowadays and to the reasonable dimensions of ships it is enough by applying four loops of the algorithm. Nevertheless, most of the vessels appear in the second and the third scale, even if their presence is transmitted over higher scales.

A detailed example with a $128 \times 128$ pixels RADARSAT image is presented in Fig. 11.

After applying one iteration of the OCWT to the input image with a ship in its centre, three wavelet components are obtained, with the same dimensions, see Fig. 12. Fig. 12a highlights horizontal discontinuities of the input image; Fig. 12b does the same for vertical irregularities whereas Fig. 12c corresponds to a low pass filtered version of the original image. Even if the presence of the ship is still appreciable in each of these components separately, none of them facilitates the detection compared to the input image. However, by combining them with a spatial product, the distribution corresponding to the background sea, uncorrelated between wavelet subbands, and that of the targets are considerably separated, as observed in Fig. 13, rendering easier (trivial in most of the cases) final decision step.
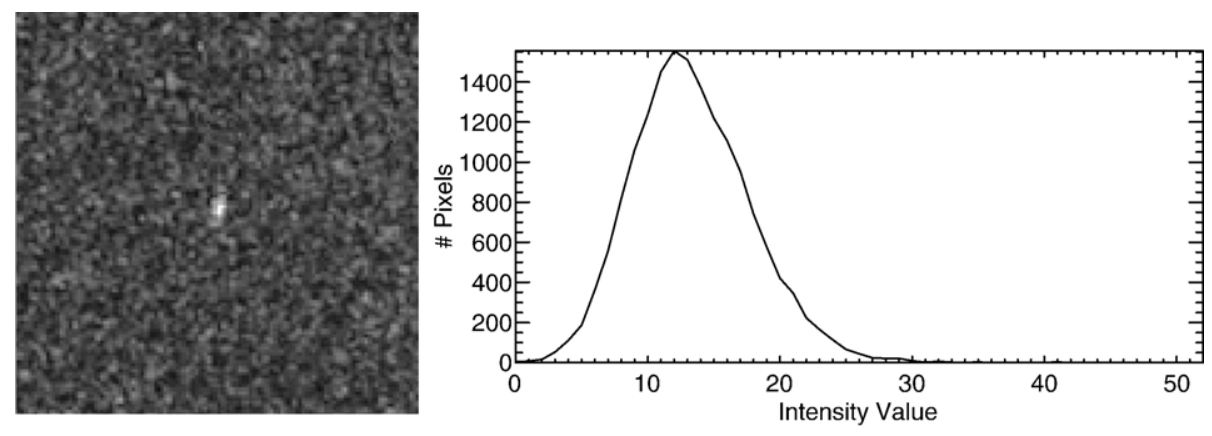

Fig. 11. RADARSAT image with its corresponding histogram. 


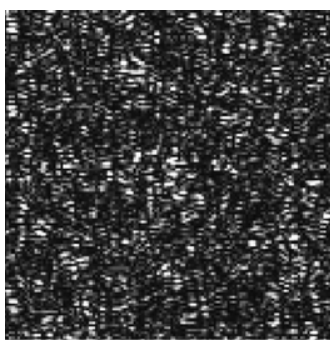

(a)

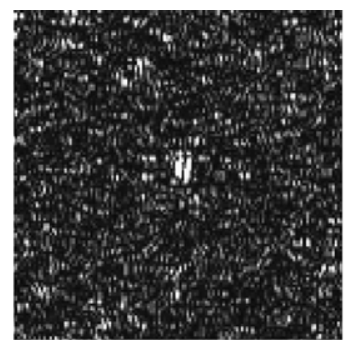

(b)

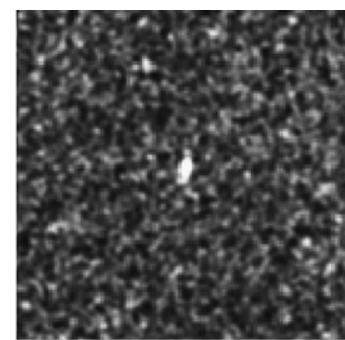

(c)

Fig. 12. Resulting wavelet subbands. (a) Wavelet component corresponding to horizontal edges, (b) wavelet component corresponding to vertical edges, (c) low pass component.

In order to evaluate the goodness of the algorithm proposed for automatic spot detection, its most interesting properties will be first analyzed and discussed. After this, a practical validation of the method is performed by applying it to RADARSAT and ENVISAT oceanic images. Unless specified, the resulting images shown correspond directly to the spatial product of the wavelet subbands: no threshold has been applied, i.e. the images are not binarized.

\subsection{Particular properties of the algorithm}

\subsubsection{Preservation of the spatial resolution}

The OCWT does not require the subsampling step of the conventional discrete WT in spite of higher redundancy and consequently, there is no degradation of the original resolution. Therefore, even smaller vessels can be detected (Fig. 14).

\subsubsection{Vessel to clutter contrast enhancement}

The most noticeable effect of the algorithm is the enhancement of contrast reached between the target and its surroundings. Since this separation of distributions is directly related to the success of the final decision step, a parameter, called significance, and denoted by, $s$ is de- fined in order to estimate quantitatively the impact of the proposed technique.

$$
s=\frac{x_{\text {target }}-\hat{x}_{\text {background }}}{\sigma_{\text {background }}}
$$

where $x_{\text {target }}$ stands for the peak intensity of the target, $\hat{x}_{\text {background }}$ stands for the value of the background mean and $\sigma_{\text {background }}$ is the background standard deviation.

Some illustrative examples are shown in Figs. 15 and 16 with their corresponding histograms. Moreover, in order to image the difficulty of deciding the way of setting the threshold, inversely related to the capabilities of detection, a gray square has been superimposed to the histogram representing the region in which the application of a threshold will provide a correct result: detection of the central target with no false alarms. Hence, a wider coloured zone means a higher flexibility in the application of the threshold and so larger possibilities of success in the automatic detection.

\subsubsection{Statistical distribution of the clutter invariant in the wavelet domain \\ It has been previously stated that the most reasonable way of facing the interpretation of SAR data is by}
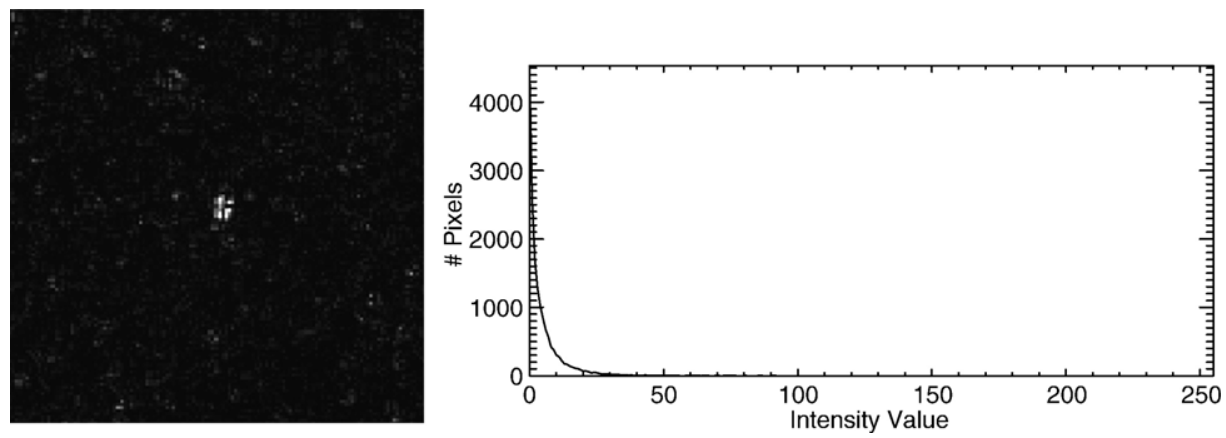

Fig. 13. Result of the application of the algorithm to the image in Fig. 11 and corresponding histogram (direct result, no threshold applied). 

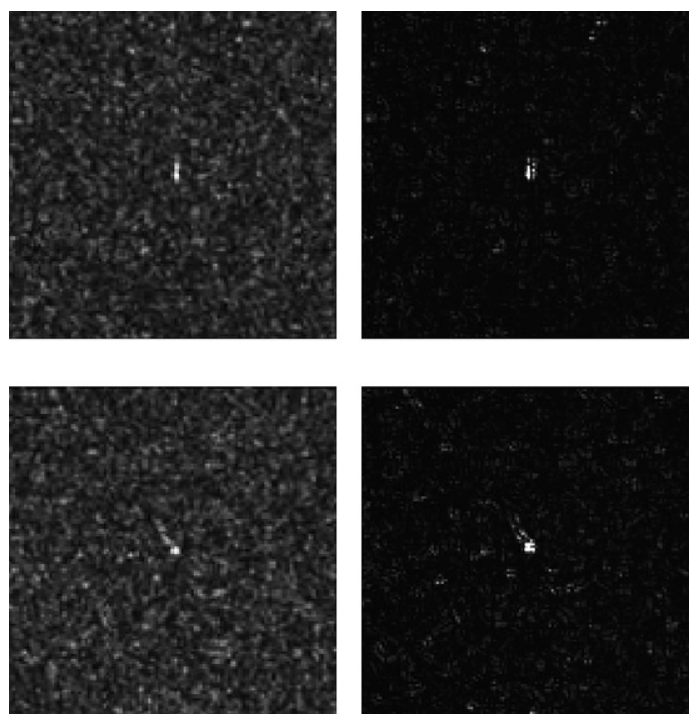

Fig. 14. Two examples of application of the algorithm to the detection of small targets (direct result, no threshold applied) in a RADARSAT image, SGF mode, HH polarization, acquired in June 2005.

assuming that SAR images are realizations of some underlying random variable. Therefore, the research of the most adequate analysis tools requires the determination of the statistical characteristics of these underlying random processes. In fact, the knowledge of the pdf makes the intrinsic randomness of the problem more manageable. Nevertheless, the great diversity of natural scenarios observed by the SAR sensors, translated into images with different statistical characteristics, makes the setting of automatic algorithms very problematic. Thus, an interesting property of the algorithm is that it brings any statistical distribution of the input data to one which is always of the same type at the output. Hence, decision rules, associated to the evaluation of a threshold, are easier.

It is demonstrated through extensive modelling of real data that the subband decompositions have significantly non-Gaussian statistics that are best described by families of heavy-tailed distributions such as the alpha-stable family (Mallat, 1999). More specifically, it is usually assumed for the wavelet coefficients that they follow an exponential pdf $f_{x}(x)$ of the form

$f_{x}(x)=K \exp \left[-\left(\frac{|\sqrt{x}|}{\alpha}\right)^{\beta}\right]$

with

$K=\frac{\beta}{2 \alpha \Gamma(1 / \beta)}$ and

$\beta=F^{-1}\left(\frac{\text { mean }^{2}}{\text { variance }}\right)$

where

$F(x)=\frac{\Gamma(2 / x)}{\Gamma(3 / x) \Gamma(1 / x)}$

In the algorithm proposed in this paper, after the wavelet decomposition, a spatial product is applied. Let us consider $z=x y$ and let us assume $x$ and $y$ independent random variables with the same distribution, then the pdf of the resulting coefficients follows

$f_{z}(z)=\int_{-\infty}^{+\infty} \frac{1}{|x|} f_{x y}\left(x, \frac{z}{x}\right) \mathrm{d} x$.

As $x$ and $y$ are independent

$f_{x y}(x, y)=f_{x}(x) f_{y}(y)$

Moreover, $x$ and $y$ have the same statistical distribution. Then:

$f_{z}(z)=\int_{-\infty}^{+\infty} \frac{1}{|x|} f_{x}(x) f_{x}\left(\frac{z}{x}\right) \mathrm{d} x$

Finally:

$f_{z}(z)=\int_{-\infty}^{+\infty} \frac{1}{|x|} K \exp \left[-\left(\frac{|x|}{\alpha}\right)^{\beta}\right] K \exp \left[-\left(\frac{|z / x|}{\alpha}\right)^{\beta}\right] \mathrm{d} x$

$f z(z)=K^{2} \int_{-\infty}^{+\infty} \frac{1}{|x|} \exp \left[-\left(\frac{|x|}{\alpha}\right)^{\beta}-\left(\frac{|z / x|}{\alpha}\right)^{\beta}\right] \mathrm{d} x$

So, even if the resulting formula is not very suggestive, it turns out that the final image has always the same nature of statistical distribution (Eq. (20)), independently from the type of statistical distribution of the input image. This makes the algorithm more robust to heterogeneities than conventional approaches which assume beforehand a particular statistical distribution of the input, as it can be seen in the example given in Fig. 17. Moreover, the subsequent decision is easier to be automatically adjusted.

\subsubsection{Capability of managing discontinuities at different scales}

The technique proposed has a multiscale capability, i.e. it can focus on different elements at different scales. 


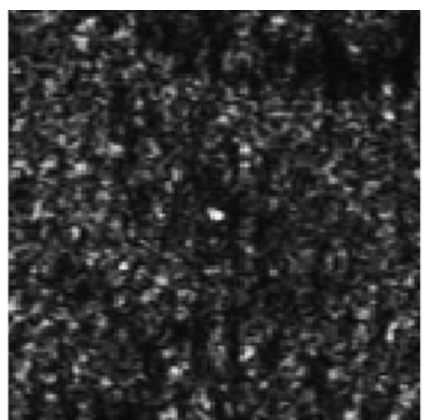

$s=6.3$

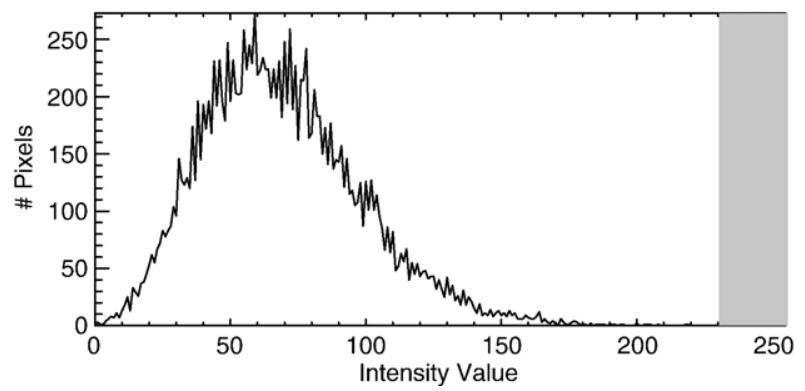

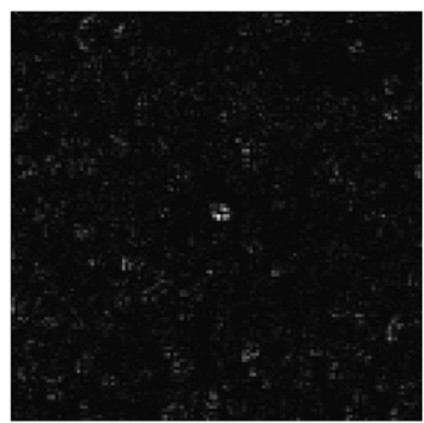

$s=24.3$

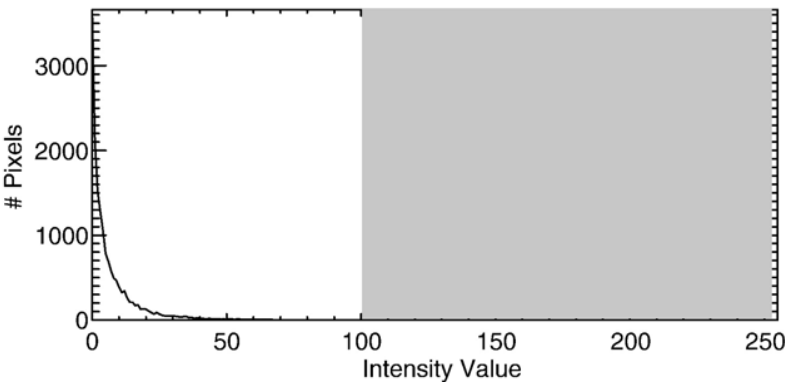

Fig. 15. Enhancement of contrast performed by the proposed algorithm. First row: original image (ENVISAT ASAR image, IM mode, VV polarization, acquired in May 2004) and result (direct result, no threshold applied). Second row: corresponding histograms with the region of applicability of a successful threshold.

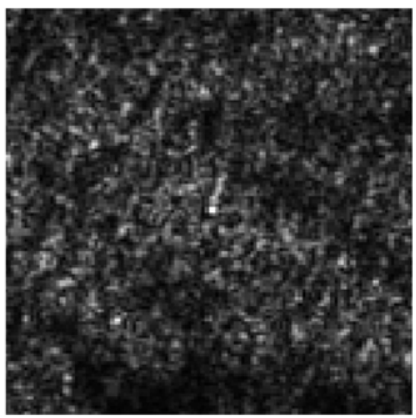

$s=5.9$

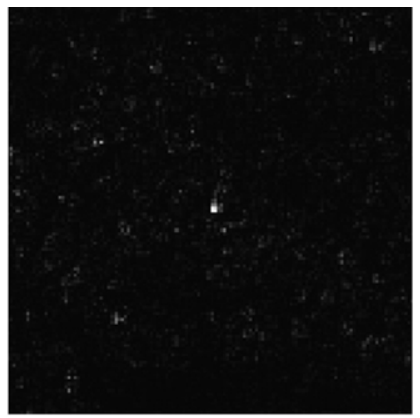

$s=24.2$

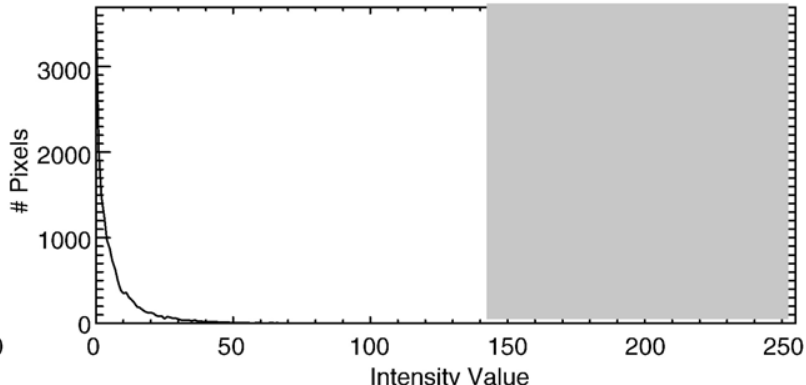

Fig. 16. Enhancement of contrast performed by the proposed algorithm. First row: original image (ENVISAT ASAR image, IM mode, VV polarization, acquired in May 2004) and result (direct result, no threshold applied). Second row: corresponding histograms with the region of applicability of a successful threshold. 


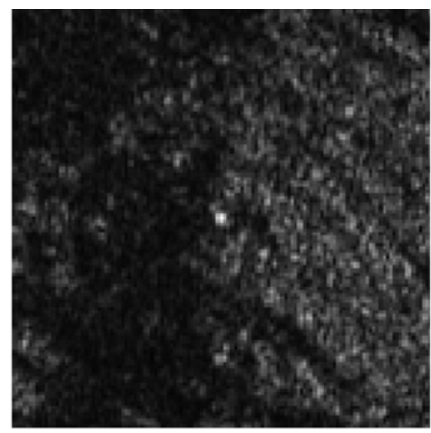

$s=5.69$

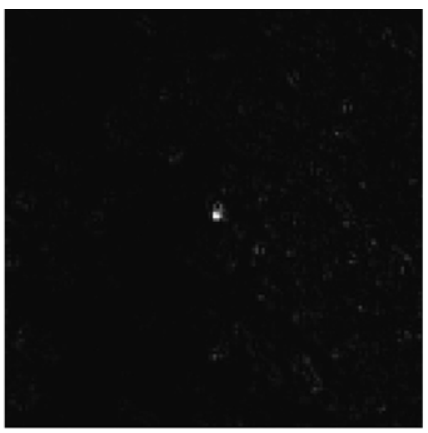

$s=40.3$
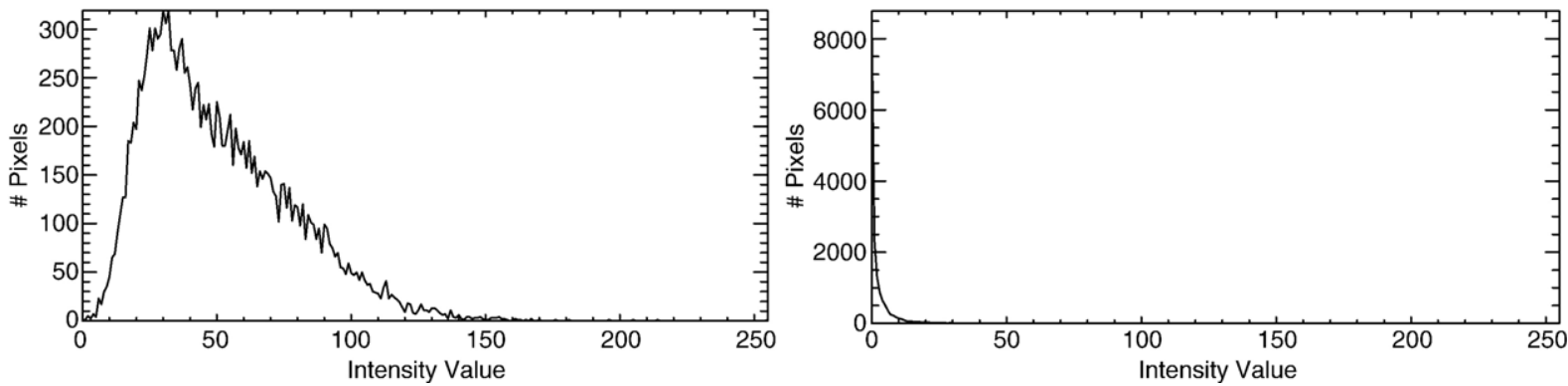

Fig. 17. Application of the proposed algorithm to an heterogeneous scene (RADARSAT image, SGF mode, HH polarization, acquired in September 2001). First row: original image and result (direct result, no threshold applied). Second row: corresponding histograms.

This means, for example, that if the method is looking for ships, which are supposed to be less than $500 \mathrm{~m}$ long, and are then expected to appear in the lower scales after 3 or 4 iterations, a big rock in the middle of the ocean would not produce a positive even if its signature is very bright in the SAR image since it will appear at higher scales, not taken into account for the purpose of ship detection. As a consequence, the algorithm proposed can intelligently manage discontinuities, usually source of false alarms.
Moreover, the technique proposed is also sensitive to a geometrical aspect in such a way that the spatial constructive coincidence of maxima only happens if the structure is localized. Therefore, other types of structures, as for example elongated ones (e.g. ice shelves) would not disturb spot detection, even if their response to the radar is very intense. An example in which a ship appears very close to ice is shown in Fig. 18. The maximum value of reflectivity of the image is on the ice surface. As a consequence, this particular situation of

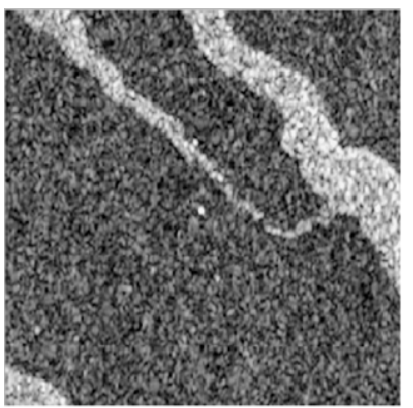

$s=2.93$

a)

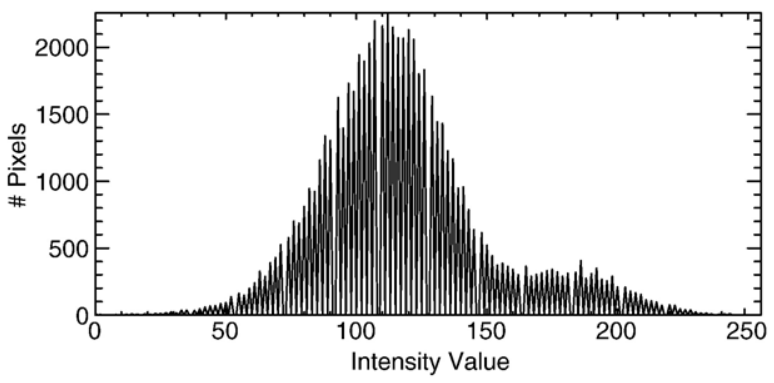

b)

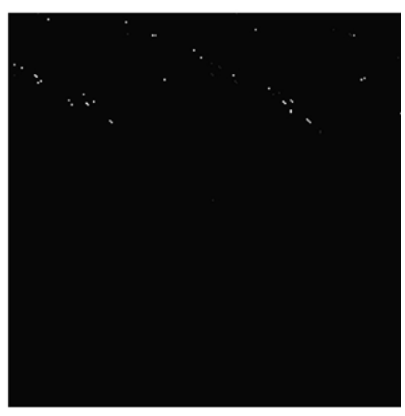

c)

Fig. 18. Application of the algorithm to an heterogeneous scene. (a) Original image (RADARSAT image, SCN mode, HH polarization, acquired in January 2003). (b) Corresponding histogram. (c) Result of the direct application of a threshold. 

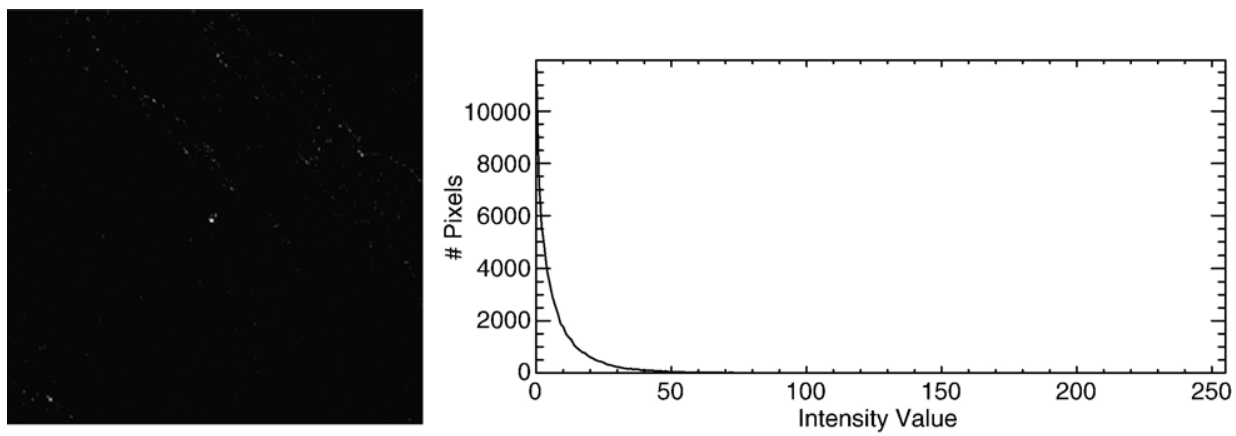

$s=24.0$

Fig. 19. Result of the application of the proposed algorithm to the image in Fig. 18 (direct result, no threshold applied) and corresponding histogram.

detection cannot be solved directly by the application of a threshold. To illustrate this point, a threshold whose value is the maximum intensity of the target has been applied. The resulting binarized image exhibits a lot of false alarms which cannot be removed since an augmentation of the value of the threshold is accompanied by a loss of the target. On the contrary, after the application of the proposed algorithm based on the OCWT, in this example, the target becomes the maximum value in the image and it is then possible to choose the value of the threshold among a large span of values leading to a correct detection: positive for the central target and absence of false alarms, see Fig. 19.

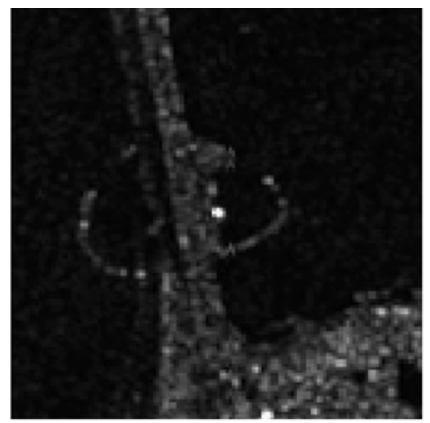

$s=8.64$

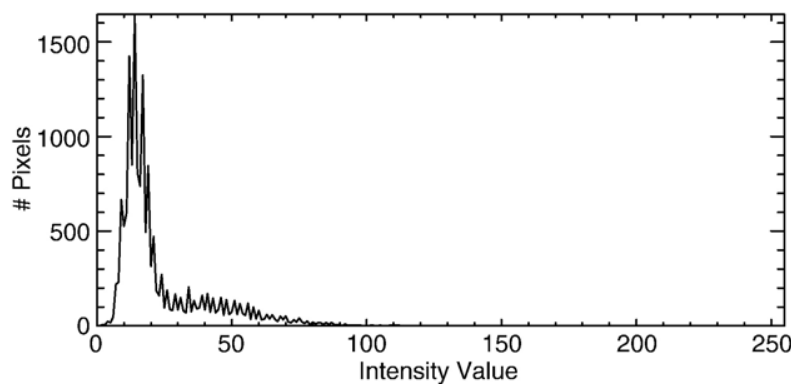

This property is particularly useful for the treatment of the region near to the coast, which is particularly critical and which is subject to increasing stress from different agencies. Conventional ship detection algorithms include a first step which consists on a land masking of the input image. This is a quite awkward process which requires the availability of precise and actualized maps. In fact, ship detection rates are considerably lower in the near-shore region than in the open sea (Greidanus, 2005).

Of course, robustness of the algorithm to discontinuities, see Figs. 19, 20 and 21, does not prevent from using a land mask since spots can also be found in the

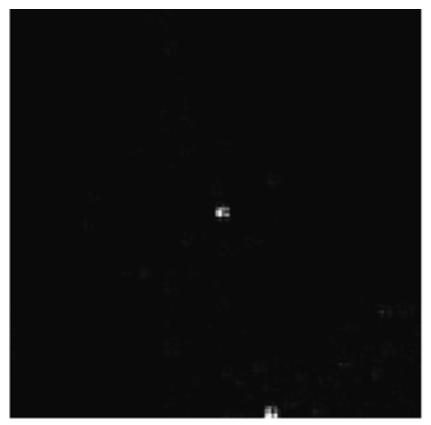

$s=49.9$

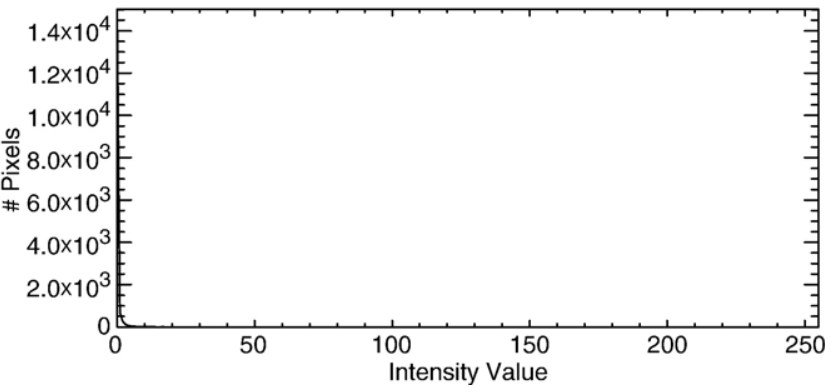

Fig. 20. Result of the application of the algorithm in a harbour (RADARSAT image, SGF mode, HH polarization, acquired in June 2005). First row: original image and result (direct result, no threshold applied). Second row: corresponding histograms. 


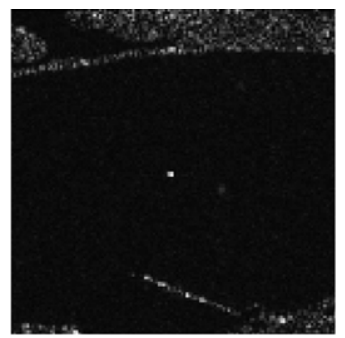

$s=8.9$

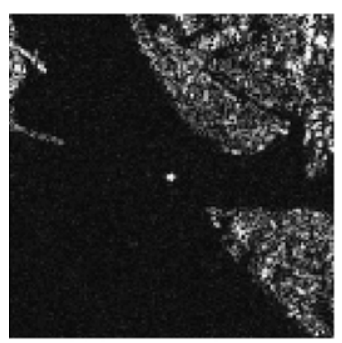

$s=11.0$

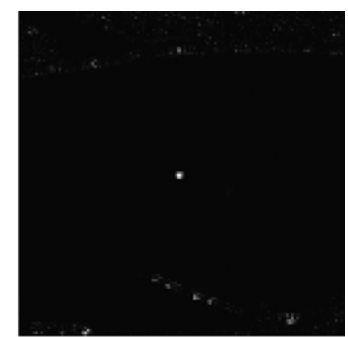

$s=37.0$

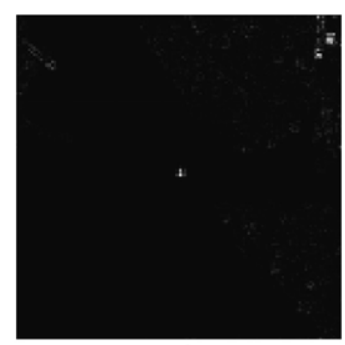

$s=49.7$
Fig. 21. Two examples of application of the algorithm in a harbour (direct result, no threshold applied). RADARSAT image, SGF mode, $\mathrm{HH}$ polarization, acquired in October 2004.

land, even more densely than in the sea. Nevertheless, detection in near shore waters is less problematic.

\subsubsection{Detection independent of the intensity}

The algorithm presented in this paper does not exclusively depend on the intensity of the targets' signature; it also depends on the local spatial correlation. As a consequence, the algorithm can detect weak targets with low reflectivities. In fact, this high sensitivity of the proposed algorithm can be regarded as a possible disadvantage. Nevertheless, this technique has been conceived in the belief that the critical issue in this kind of application is detection, rather than false alarms.

\subsection{Validation}

The method presented in this paper has been implemented to be able to efficiently process complete SAR images of different types. A final threshold step has been added after the application of the algorithm based on the WT. A text file is then obtained with the list of positions of eventual targets. This procedure is completely unsupervised and it takes few minutes, depending on the dimensions of the image. Two examples of processing are presented hereafter. In order to test the performance of the algorithm proposed, the positions obtained have been compared to ground truth data (when available) and to visual inspection. Moreover, results have also been correlated to the detections of other automatic and operational algorithms tested on the same images within the scope of the DECLIMS project (Greidanus et al., 2004). All of those operational systems rely on the detection of bright pixels compared to the local background (CFAR approach), sometimes employing a template to match expected target shape. One of the main findings of the DECLIMS project was that the operational algorithms, although performing markedly different on individual images, had an overall comparable performance.

\subsubsection{Example of operation on a RADARSAT image}

The first example of analysis corresponds to a $8892 \times 8711$ pixels $\mathrm{SGF}$ mode RADARSAT image, acquired in July 2002 in the Cantabrico area, Northern Spain (see Fig. 22). The resolution is about $22 \mathrm{~m}$ in range and $27 \mathrm{~m}$ in azimuth. This image has been selected because the region usually presents a high traffic of fisheries. Vessels in this region are usually very small and made of wood so that most of them are difficult to detect.

After running the algorithm proposed based on the WT, 103 possible targets were detected. The specific distribution of these positives is as follows:

- 22 targets detected by other automatic algorithms and corresponding quite probably to vessels, 5 of them reported their VMS position.

- 2 targets not detected by other automatic algorithms but with a reported VMS position, as shown in Fig. 23.

- 56 targets not detected by other automatic algorithms and corresponding quite probably to vessels. These targets have been considered as vessels by means of visual inspection. Some representative examples are shown in Fig. 24.

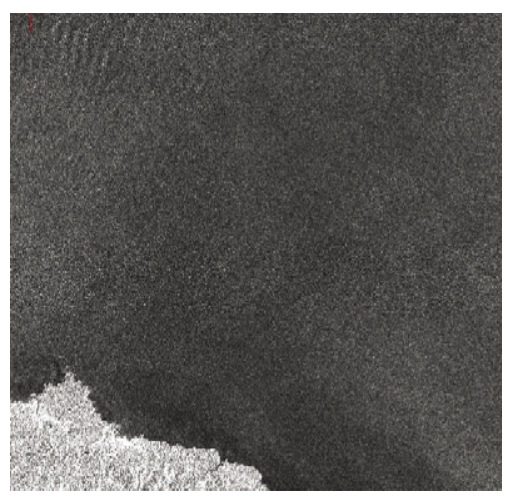

Fig. 22. RADARSAT image of the Cantabrico area, Northern Spain. 


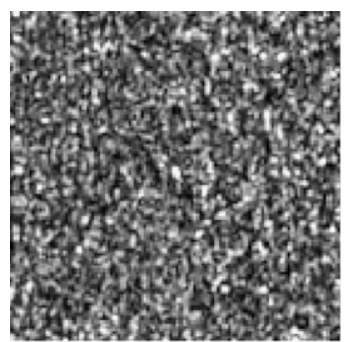

$$
s=4.5
$$

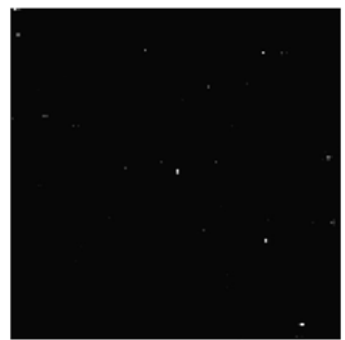

$s=13.0$

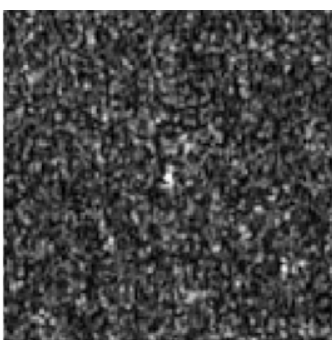

$s=7.7$

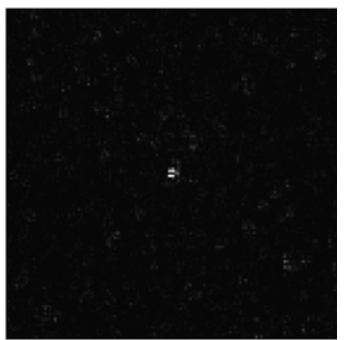

$s=33.6$
Fig. 23. Positives (direct result, no threshold applied) validated through VMS positions.

- 23 targets not detected by other automatic algorithms and visual inspection cannot decide whether they may correspond to ships signatures or not. Some representative examples of this category are shown in Fig. 25. It may be interesting to notice that, even if a decision is not possible, the automatic algorithm is performing correctly since each positive is due to the presence of a spot in the original image. Moreover, these spots could reasonably constitute signatures of ships. In fact, two examples of signatures certainly belonging to ships (as VMS ground truth was available) can be seen in Fig. 23 and they are similar to the examples shown in this one.

Additionally, an extensive visual inspection did not find any other possible target not detected by the proposed algorithm.

\subsubsection{Example of operation on an ENVISAT image}

The second example of analysis corresponds to a $4641 \times 8513$ pixels ENVISAT ASAR IMP image acquired in July 2004, see Fig. 26. The resolution is about 30 both in range and in azimuth.

In this image, 100 targets were detected by the algorithm proposed in this paper. Similarly, the specific distribution of these positives is as follows:

- 66 targets detected by other automatic algorithms and corresponding quite probably to ships signatures.

- 26 targets not detected by other automatic algorithms and corresponding quite probably to ships signatures. Some illustrative examples are shown in Fig. 27.

- 8 targets not detected by other automatic algorithms and visual inspection cannot decide whether they may correspond to ships signatures or not. Some illustrative examples are shown in Fig. 28.

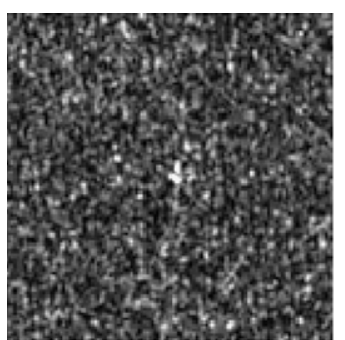

$s=6.3$

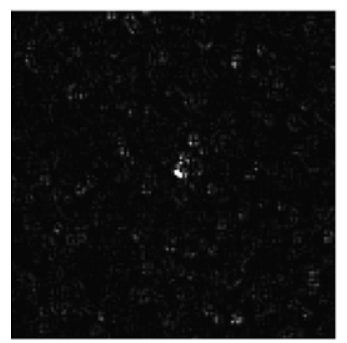

$s=37.4$

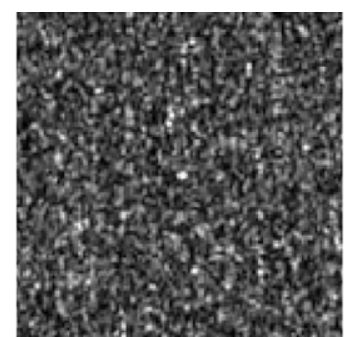

$s=6.3$

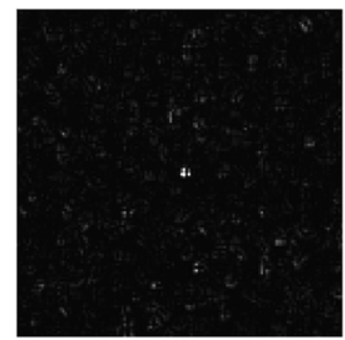

$s=40.9$

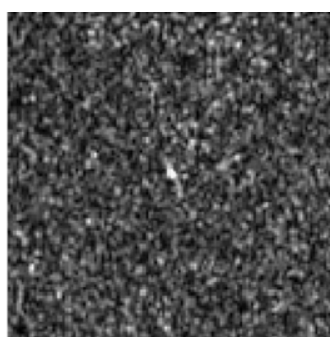

$s=6.7$

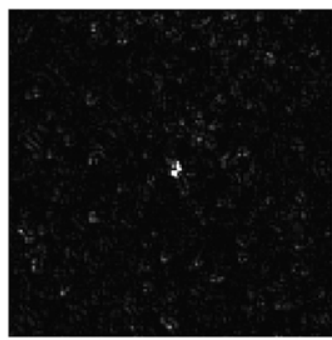

$s=33.4$

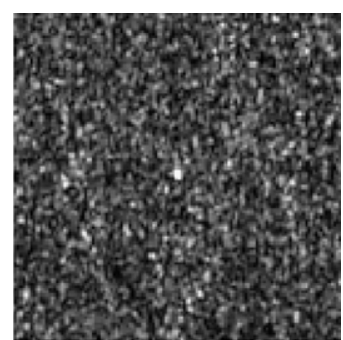

$s=6.5$

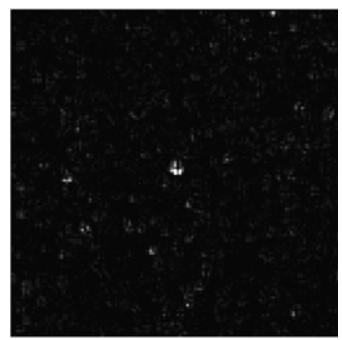

$s=40.4$

Fig. 24. Four representative examples (direct result, no threshold applied) of positives corresponding probably to ships. 


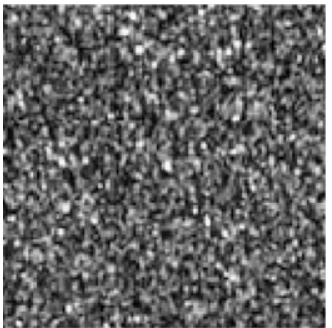

$s=5.7$

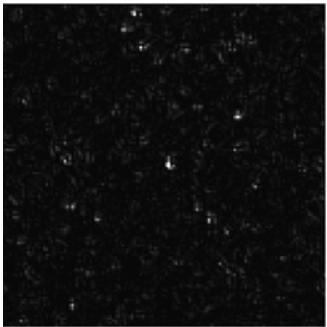

$s=36.2$

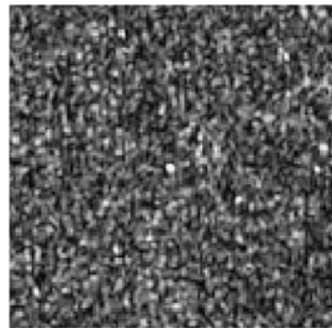

$s=6.8$

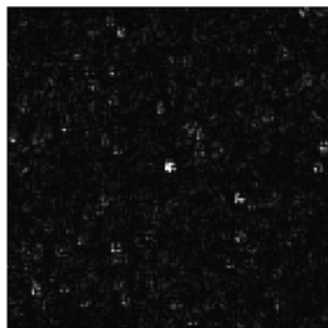

$s=31.7$

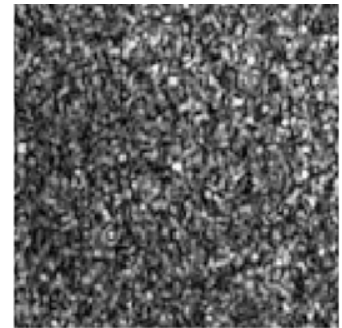

$s=6.2$

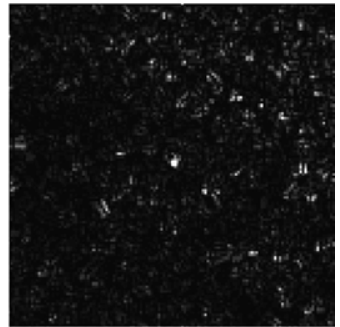

$s=21.0$

Fig. 25. Three representative examples (direct result, no threshold applied) of positives in which visual inspection could not decide whether they correspond to a possible target or not.

Additionally, the visual inspection of the image did not find any other possible target not detected by the proposed algorithm.

\section{Extension to multidimensional data}

As presented in the previous sections, the particular properties of the data provided by SAR systems, in conjunction with advanced signal processing techniques, make possible to define a robust technique for ship detection, both in coastal areas and in the open ocean. Nevertheless, the important impact of the SAR techniques in remote sensing must be found in the fact that multidimensional SAR configurations are possible. These new configurations are basically designed to exploit the correlation structure among the different SAR images, making possible to retrieve quantitative physical information (Cloude and Pottier, 1996). Despite this correlation structure is not taken into account, it will be shown in the following that the availability of multiple SAR images of the same area can improve the performance of the detection algorithm presented in the previous sections.

SAR interferometry (InSAR) is based on acquiring two SAR images from slightly different positions in space (Bamler and Hartl, 1998). This diversity in space allows retrieving information about the vertical structure of the surface, so that topographic information can be derived. In the case of space borne SAR systems, this multidimensional configuration is not useful for the ship detection problem since SAR images are acquired at different times. Since this difference may range from days to months, obviously, the two SAR images would not contain the same ships. As a consequence, both SAR images cannot be jointly considered to improve ship delectability. Polarimetric SAR systems (PolSAR) represent a second type of multidimensional SAR system configuration, in which the diversity is obtained by

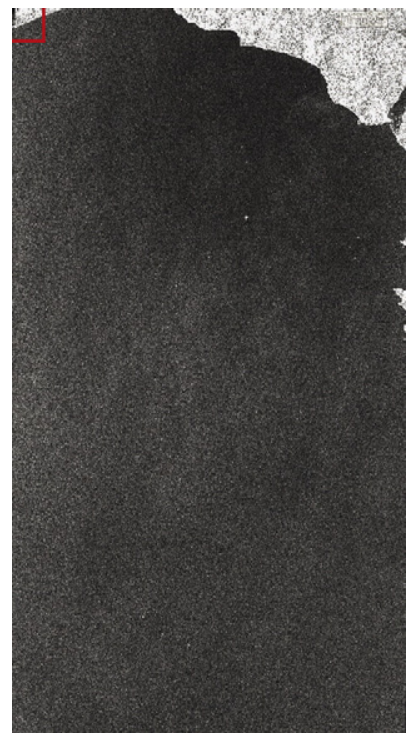

Fig. 26. ENVISAT image. 


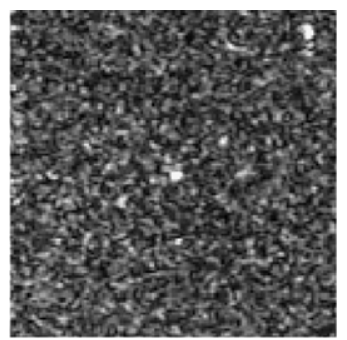

$s=9.2$

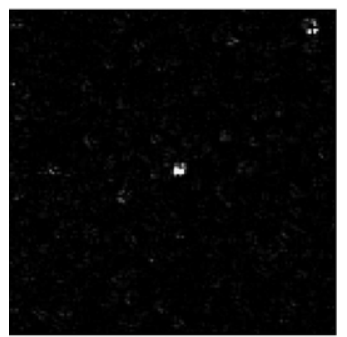

$s=36.0$

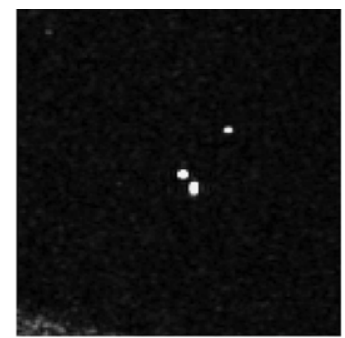

$s=29.7$

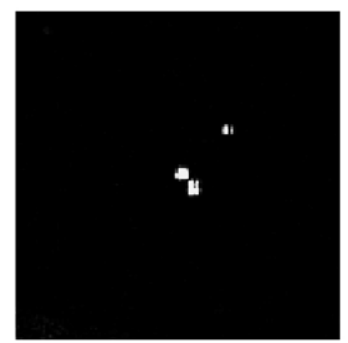

$s=47.5$

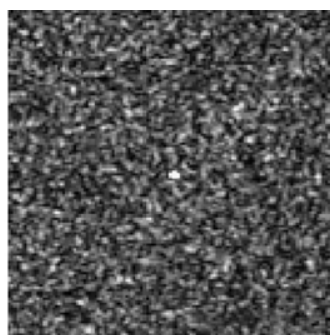

$s=8.8$

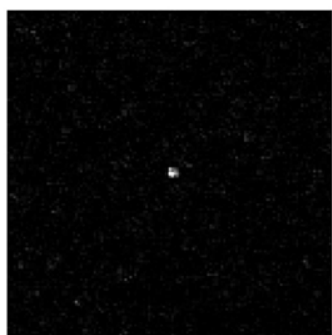

$s=44.7$

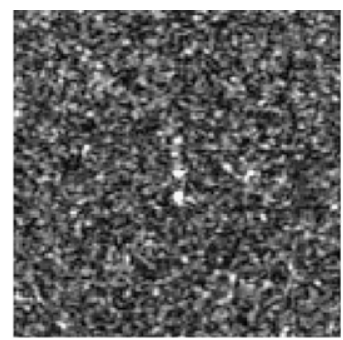

$s=8.0$

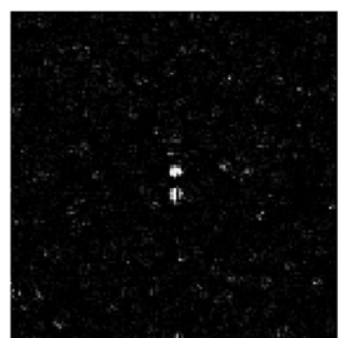

$s=38.8$

Fig. 27. Four representative examples (direct result, no threshold applied) of positives corresponding probably to ships.

considering transmitted and received echoes at different electromagnetic field polarizations (Ulaby and Elachi, 1990). The most usual configuration of this type of fully polarimetric SAR systems is a monostatic SAR, where the transmitter and the receiver are located at the same spatial position. In this case, the PolSAR system records three SAR images at the same time. Nevertheless, currently there is no fully polarimetric data of spaceborne
SAR systems available, although some future missions are planned as the Canadian RADARSAT 2 and the German TerraSAR X or, as the Japanese ALOS system that is already in the commissioning phase.

The only current system which can be considered to test the ship detection improvement by means of multiple SAR images is the European ENVISAT ASAR system in its alternating polarization (AP) operation

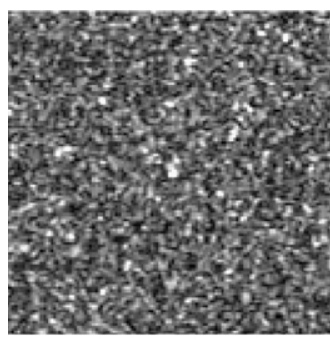

$s=6.1$

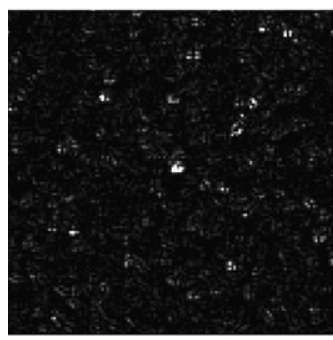

$s=28.0$

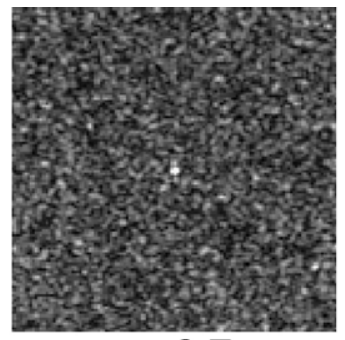

$s=8.7$

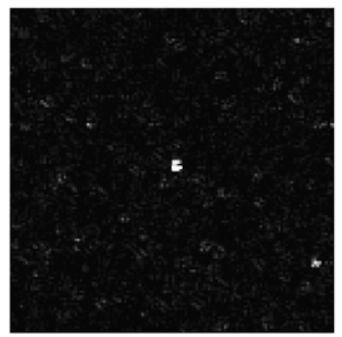

$s=40.5$

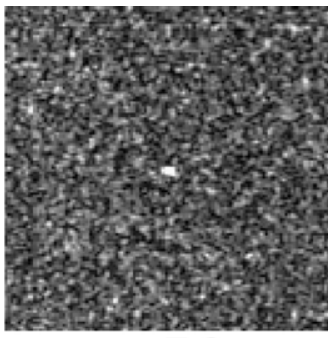

$s=7.3$

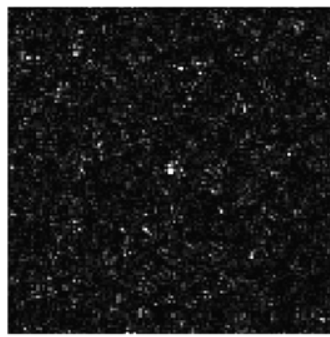

$s=22.5$

Fig. 28. Three representative examples (direct result, no threshold applied) of positives in which visual inspection could not decide whether they correspond to a possible target or not. 


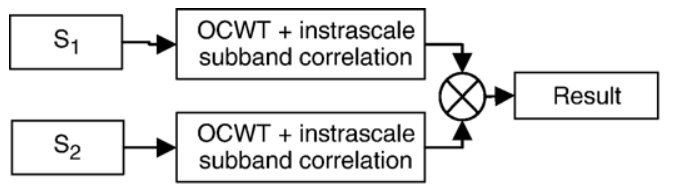

a)

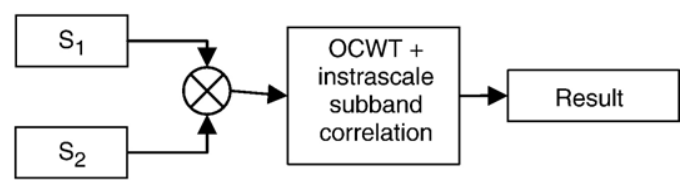

b)

Fig. 29. Ship detection algorithms with PolSAR data. (a) Algorithm based of the spatial product of the single SAR imagery detection results. (b) Algorithm based on performing ship detection on the spatial product of SAR images. $\mathrm{S}_{1}$ and $\mathrm{S}_{2}$ denote every one of the SAR images considered by the detection algorithms.

mode. In this mode, ASAR is not able to provide fully polarimetric information, but partial polarimetric information (Souyris et al., 2005), i.e., the system records two instead of three SAR images. Although the complete polarimetric information about the scatterer of interest cannot be retrieved, utilizing both SAR images shows nevertheless great potential to improve ship detection. Two approaches accounting for this issue will be outlined in the following.

The first point to consider when multiple SAR images are available is how to combine them in such a way that ship detection is improved. The algorithm detailed in the previous sections employs the spatial product of the different components of the wavelet transform of the input SAR image, based on the assumption that ship locations are not altered by the transform itself. Since PolSAR systems acquire the images at the same instant of time, one can extend the previous idea to the spatial product of the different SAR images. As depicted in Fig. 29, two algorithms have been developed based on this extension. They mainly differ in the stage of processing, at which the two (or even more) images are combined.

Both algorithms have been applied to several ENVISAT datasets acquired in AP mode. Based on the parameter of significance, see Eq. (11), it has been found that ship detection performance of both approaches is quite similar. Therefore, considering the lower computational cost, the second algorithm has been selected since it applies the ship detection algorithm only once to the result of the spatial product of the different images. The results of this approach are visualized in Figs. 30 and 31. The first conclusion which can be drawn from both figures is the expected result that the use of multiple SAR images improves ship detection. If one compares Fig. 30f with $\mathrm{d}$ and $\mathrm{e}$, it is

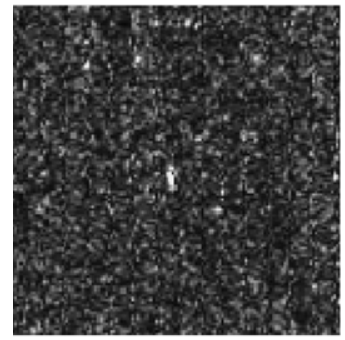

a) $s=10.4$

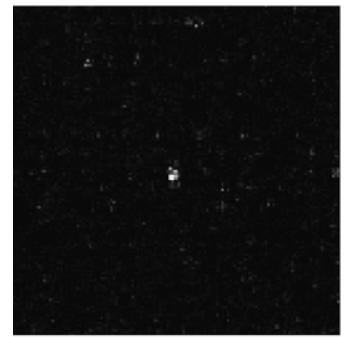

d) $s=33.8$

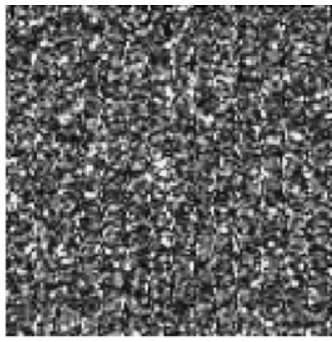

b) $s=6.46$

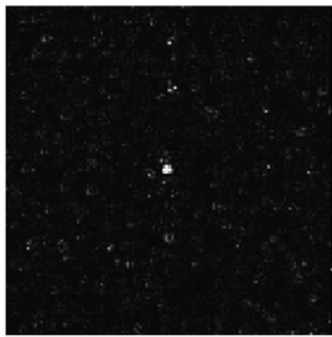

e) $s=27$

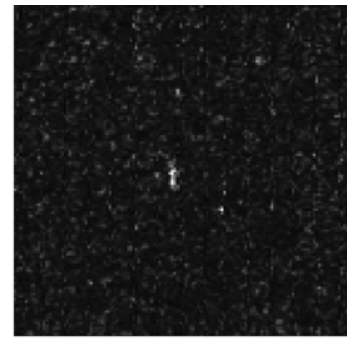

c) $s=20.6$

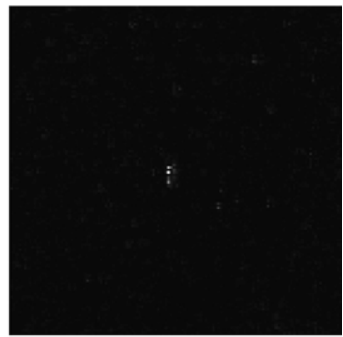

f) $s=70.9$

Fig. 30. (a) First SAR image HH pol., (b) second SAR image HV pol., (c) spatial product of SAR images, (d) ship detection applied of the image (a), (e) ship detection applied of the image (b), (f) ship detection applied to the spatial product of SAR images. 


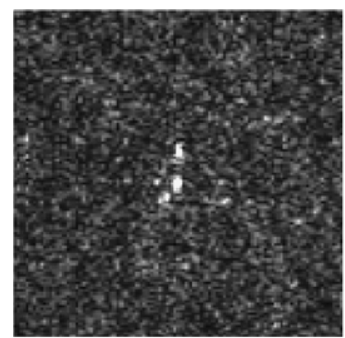

a) $s=10.6$

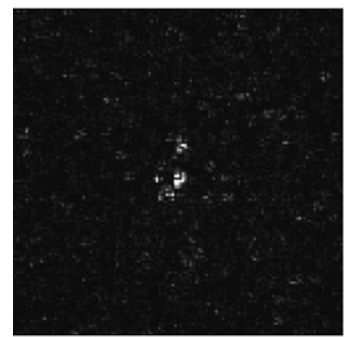

d) $s=25.7$

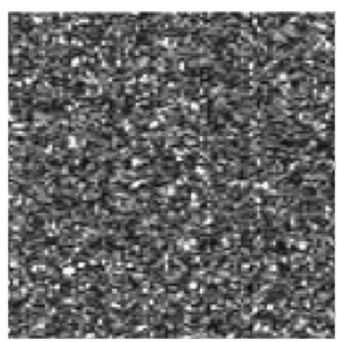

b) $s=-1.07$

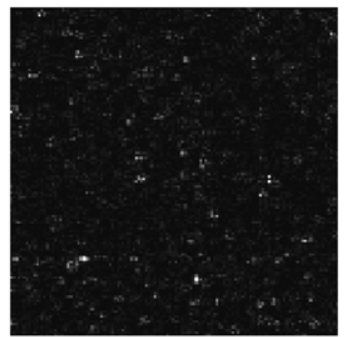

e) $s=0$

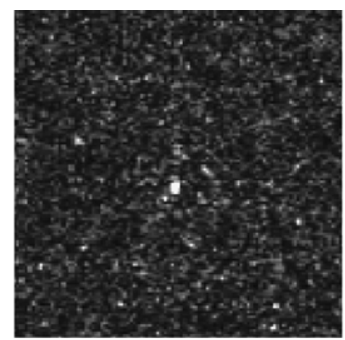

c) $s=11.9$

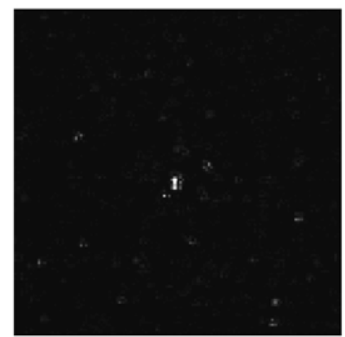

f) $s=55.3$

Fig. 31. (a) First SAR image HH pol., (b) second SAR image HV pol., (c) spatial product of SAR images, (d) ship detection applied of the image (a), (e) ship detection applied of the image (b), (f) ship detection applied to the spatial product of SAR images.

possible to observe that the proposed algorithm is able to double the significance parameter, which implies a better detection performance and a decrease of the false alarm rate.

Since PolSAR data is sensitive to the geometric properties of the target under investigation (depending on the particular position of a ship with respect to the imaging system) some ships may not appear in one of the SAR images. This situation is for instance visible in Fig. 31. As a result, the ship would be only detected in the first SAR image. Nevertheless, this situation can be still exploited with the proposed algorithm for PolSAR data. Although the ship is not visible in the second SAR image, the information of this image can be exploited in order to reduce the effect of the clutter on the first SAR image. Again, one may observe that the proposed algorithm is able to double the detection capability, even in awkward situations.

The results provided by the algorithms presented and analyzed in this section lead to the conclusion that, even under the limitations of the ENVISAT AP imaging mode regarding the completeness of polarimetric information, this imaging mode can be successfully exploited to improve ship detection. Although the presented algorithms have been applied to only two polarimetric SAR images, they can be easily extended to fully polarimetric SAR or even to other multidimensional SAR system configurations.

\section{Conclusions}

After justifying the use of a time-frequency framework for the exploitation of SAR images and in particular for ship detection purposes, an alternative automatic technique to CFAR methods has been presented. The algorithm proposed appears to be simple, robust and reliable. It is not exclusively dependent on the intensity and therefore it is able to detect weak targets. Moreover, its multiscale capability makes it useful to intelligently deal with complex clutter situations and for the automatic treatment of near shore waters. The algorithm has been extended to multidimensional data and it has been shown that the availability of polarimetric diversity augments the automatic detection capabilities.

The results obtained confirm that spaceborne SAR systems, jointly with a set of specific algorithms for a reliable and quick interpretation of SAR data can constitute an extremely valuable tool to assist authorities in monitoring vessel traffic.

\section{Acknowledgements}

This work has been funded by the Spanish MCYT under the project TEC2005-06863-C02-01. The authors would like to thank the DECLIMS and the IMPAST projects for providing access to the SAR images database and VMS positions. 


\section{References}

Arnesen, T.N., Olsen, R.B., 2004. Literature Review on Vessel Detection, FFI/RAPPORT-2004/02619. Norwegian Defence Research Establishment (FFI), Kjeller, Norway.

Bamler, R., Hartl, P., 1998. Synthetic aperture radar interferometry. Inverse Problems 14 (4), R1-R54.

Cloude, S.R., Pottier, E., 1996. A review of target decomposition theorems in radar polarimetry. IEEE Transactions on Geoscience and Remote Sensing 34 (2), 498-518.

Cornaert, M., deGroof, H., Hubbard, N., Levy, E., 2001. Key elements of the GMES EC Draft Action Plan, GMES, Brussels 2001. Also available at http://www.gmes.info/library/files/Reference\%20Documents/ GMES_EC_Action\%20plan_Brief.pdf (Accessed September 18, 2006).

Curlander, J.C., McDonough, R.N., 1991. Synthetic Aperture Radar: Systems and Signal Processing. John Wiley \& Sons, Inc., New York.

Ferreti, A., Prati, C., Rocca, F., 2001. Permanent scatterers in SAR interferometry. IEEE Transactions on Geoscience and Remote Sensing 39 (1), 8-20.

Flandrin, P., 1992. Wavelet analysis and synthesis of fractional Brownian motion. IEEE Transactions on Information Theory 38 (2-2), 910-917.

Goodman, J.W., 1976. Some fundamental properties of speckle. Journal of Optical Society of America 66 (11), 1145-1149.

Greidanus, H., 2005. Assessing the operationality of ship detection from space. Proceedings of EURISY - New space services for maritime users, Paris, France, on CD-ROM.

Greidanus, H., 2006. Findings of the DECLIMS project - detection and classifications of marine traffic from space. Proceedings of SEASAR 2006, Frascati, Italy, on CD-ROM.

Greidanus, H., Clayton, P., Indregard, M., Staples, G., Suzuki, N., Vachoir, P., Wackerman, C., Tennvassas, T., Mallorqui, J., Kourti,
N., Ringrose, R., Melief, H., 2004. Benchmarking operational SAR ship detection. Proceedings of IGARSS 2004, Anchorage, USA, on CD-ROM.

Law, N.F, Siu, W.C., 2003. A fast and efficient computational structure for the $2 \mathrm{D}$ over-complete wavelet transform. Proceedings of ICASSP, vol. 3, pp. 309-312.

Lee, J.S., 1981. Speckle analysis and smoothing of synthetic aperture radar images. Computer Graphics and Image Processing 17, 24-32.

Liebe, C.C., 1993. Pattern recognition of star constellations for spacecraft applications. IEEE Aerospace and Electronic Systems Magazine 8 (1), 31-39.

Mallat, A., 1999. Wavelet Tour on Signal Processing. Academic Press, New York, USA.

Souyris, J.C., Imbo, P., Fjortoft, R., Mingot, S., Lee, J.S., 2005. Compact polarimetry based on symmetry properties of geophysical media: the $\pi / 4$ mode. IEEE Transactions on Geoscience and Remote Sensing 43 (3), 634-646.

Stewart, C.V., Moghaddam, B., Hintz, K.J., Novak, L.M., 1993. Fractional Brownian motion models for synthetic aperture radar imagery scene segmentation. Proceedings of the IEEE 81 (10), $1511-1522$

Ulaby, F.T., Elachi, C., 1990. Radar Polarimetry for Geoscience Applications. Artech House, Norwood, USA.

Villasenor, J.D., Belzer, B., Liao, J., 1995. Wavelet filter evaluation for image compression. IEEE Transactions on Image Processing 4 (8), 1053-1060.

Zheng, B., Qian, W., Clarke, L.P., 1996. Digital mammography: mixed feature neural network with spectral entropy decision for detection of microcalcifications. IEEE Transactions on Medical Imaging 15 (5), 589-597. 